Check for updates

\title{
BUSINESS INTELLIGENCE + LEAN MANUFACTURING: UMA REVISÃO SISTEMÁTICA DA LITERATURA (2008-2018)
}

\author{
BUSINESS INTELLIGENCE + LEAN MANUFACTURING: \\ A SYSTEMATIC LITERATURE REVIEW (2008-2018)
}

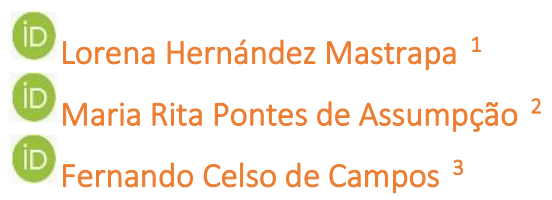

${ }^{1}$ Mestre em Engenharia de Produção Universidade Metodista de Piracicaba - UNIMEP lorenahmastrapa@gmail.com

2 Doutora em Engenharia de Produção Universidade Virtual do Estado de São Paulo UNIVESP.

ritassumpa@gmail.com

${ }^{3}$ Doutorado em Engenharia Mecânica Universidade Metodista de Piracicaba - UNIMEP fernando.campos@unimep.br

Recebido em: 09 mar. 2018

Aprovado em: 14 set. 2018

Cite como - American Psychological Association (APA)

Mastrapa, L. H., Assumpção, M. R. P. de., \& Campos, F. C. de. (2021, jan./mar.). Business Intelligence + Lean Manufacturing: uma revisão sistemática da literatura (2008-2018). Exacta, 19(1), 17-34.

https://doi.org/10.5585/exactaep.v19n1.11356.
Resumo: Lean Manufacturing (LM) é uma filosofia de gestão apoiada por um grupo de técnicas que, quando combinadas e amadurecidas, reduzem o tempo e o custo de produção, maximizam o valor ao cliente e minimiza desperdícios. Para isto a tomada de decisão desempenha um papel fundamental e se torna um ponto crítico para esta filosofia de gestão. A ferramenta Business Intelligence (BI) fornece uma abordagem baseada em dados para vincular as metas estratégicas das empresas às políticas gerenciais e ações operacionais, podendo ser de ajuda para as empresas enxutas. Assim, o presente estudo pretende analisar as principais aplicações das ferramentas de BI para dar suporte à tomada de decisão às empresas que aplicam LM. Com apoio do software de revisão sistemática StArt, foi feita uma análise da literatura atual relacionada ao tema de pesquisa. A análise permitiu definir oportunidades de aplicação de BI nas empresas que, de alguma forma, utilizam o LM.

Palavras-chave: Business Intelligence. Lean Manufacturing. tomada de decisão. revisão sistemática.

Abstract: Lean Manufacturing (LM) is a management philosophy supported by a group of techniques when combined and matured, reduce production time and cost, maximize customer value and minimize waste. For this, decision-making plays a fundamental role and becomes a critical point for this management philosophy. The Business Intelligence (BI) tool provides a data-driven approach to linking strategic business goals to managerial policies and operational action can be of great help to lean companies. Thus, the present study intends to analyze the main applications of the $\mathrm{BI}$ tools to support the decision making to the companies that apply the LM. With the support of the StArt, a systematic review software, an analysis of the current literature related to the research topic was done. The analysis allowed us to define $\mathrm{BI}$ application opportunities in companies that, in some way, use LM.

Keywords: Business Intelligence. Lean Manufacturing. Decision-making. Systematic review.

\section{(cc) BY-NC-SA}


Introdução

A produção enxuta ou Lean Manufacturing (LM) é considerada um dos paradigmas mais relevantes da indústria, capaz de aumentar a competitividade das empresas, reduzindo todas as atividades sem valor, melhorando a produtividade e a qualidade (Taddeo, Simboli, Di Vincenzo \& Ioppolo, 2019). Os pioneiros do LM desenvolveram uma grande quantidade de ferramentas e técnicas que permitem aos seguidores enfrentar vários problemas de produção, eliminar desperdícios e tornar enxutos os processos (Wan \& Chen, 2009). A maioria das ferramentas enxutas foram criadas para resolver problemas específicos, como alto nível de trabalho em processo, baixa disponibilidade de equipamentos ou longo tempo de configuração (setup).

Aplicar ferramentas de apoio à tomada de decisão para orientar os usuários durante toda a jornada para o LM é uma etapa importante da integração com a tecnologia da informação (TI). Segundo Wan \& Chen (2009), uma ferramenta de apoio à tomada de decisão é desejável para aprimorar as ferramentas de orientação para a implementação ou melhora de todo o sistema enxuto. Os profissionais de LM precisam ser capazes de avaliar o desempenho atual de seus sistemas e identificar os pontos fracos e os problemas de melhorias. Para os iniciantes, selecionar o ponto de partida mais eficiente geralmente requer mais experiência do que o que eles têm. Para os profissionais avançados, identificar as oportunidades para melhorias futuras requer uma investigação completa e um amplo conhecimento. Para a equipe de execução, informações e diretrizes pertinentes devem ser fornecidas quando um projeto de melhoria é definido. Para a administração, as melhorias precisam ser monitoradas e frequentemente comparadas com instalações semelhantes na empresa ou com concorrentes. Portanto, as informações do apoio à tomada de decisão devem cobrir as necessidades de vários aspectos dos envolvidos na implementação dos conceitos do LM.

Com o aumento da concorrência e da complexidade do meio ambiente e o rápido desenvolvimento tecnológico, a adoção de sistemas de medição de desempenho baseados em TI aumentou nos últimos anos (Vallurupalli \& Bose, 2018). Ferramentas eficazes que apoiam o processo de tomada de decisão foram desenvolvidas, por exemplo, as de Business Intelligence (BI), que segundo Haque, Derksen, Calado \& Foster (2015), fornece à empresa a capacidade de coletar, manter e organizar o seu conhecimento. O Bl emergiu como uma importante área de estudo para profissionais e pesquisadores, refletindo a magnitude e o impacto de problemas relacionados a dados presentes em organizações empresariais contemporâneas (Chen, Chiang \& Storey, 2012; Haque, Derksen, Calado \& Foster, 2015; Shollo \& Galliers, 2015). Do ponto de vista dos sistemas de informação, os sistemas de BI combinam coleta e armazenamento de dados com ferramentas analíticas para apresentar informações internas e competitivas complexas para planejadores e tomadores de decisão (Negash, 2004; Ghosh \& Scott, 2011). Além disso, o BI é uma ferramenta versátil e abrangente para análise de causalidade e análises corporativas, uma vez que fornece uma abordagem baseada em dados para vincular as metas estratégicas das empresas às políticas táticas e ações operacionais (Wang, 2016). 
A partir da ausência de pesquisas que relacionam LM com aplicações de BI surgiu o seguinte questionamento: "Como as empresas que vivenciam o LM utilizam atualmente ferramentas de BI para a tomada de decisão?"

Assim o presente estudo tem como objetivo, analisar aplicações atuais de ferramentas de BI no suporte à tomada de decisão em empresas que estão em jornada do LM.

Nas próximas seções desse trabalho são descritas a metodologia para o desenvolvimento da pesquisa, ou seja, quais os passos da revisão sistemática da literatura feita; logo depois, é analisada a literatura extraída referente às aplicações do BI para a tomada de decisão, seguido pelo referencial teórico selecionado que está relacionado com ferramentas de tomada de decisão em empresas que aplicam o sistema LM. Finalmente é feita uma análise comparativa destes estudos de aplicação com o intuito de determinar as oportunidades encontradas.

\section{Metodologia}

Segundo Pereira \& Galvão (2014), a revisão sistemática tem como objetivo apresentar a síntese da evidência disponível sobre uma questão de pesquisa, a busca e a seleção devem ser bem executadas, de modo que seja possível identificar e incluir estudos relevantes sobre o assunto. Baseado nisto, com o intuito de identificar estudos relevantes do BI no LM foi desenvolvida uma revisão sistemática da literatura (R.S.L.).

Para esta análise foi utilizado o software de R.S.L. denominado de StArt, desenvolvido pelo LAPES/UFSCAR (Fabbri et al., 2016).

Na Tabela 1 apresenta-se o protocolo seguido para desenvolver a presente R.S.L., baseado em Kitchenham \& Charters (2007), de acordo com as fases de: planejamento, execução e sumarização. O protocolo é elemento fundamental para a fase de planejamento da R.S.L.

\section{Planejamento}

Nesta fase é definido o objetivo e a questão principal da pesquisa, além das palavras chaves para criar as strings de busca nas bases de dados selecionadas e os critérios de inclusão e exclusão como apresentados na Tabela 1.

Para a busca da literatura que vinculasse o uso das ferramentas de BI ao $L M$ foram realizadas 2 tentativas nas bases de dados. Na primeira delas usando as strings de busca "Business Intelligence" e "Lean Manufactuirng" foram obtidos resultados, mas a literatura não tratava o tema pesquisado. Isto levou a segunda estratégia de busca, onde foram separados em duas strings de busca: "Business Intelligence" AND "decision support tools" e "Lean Manufactuirng" AND "decision support tools", procurando, com isto, a forma de analisar de formas separadas as aplicações de ferramentas de tomada de decisão em cada uma e, no fim, baseado nestas experiências, avaliar se foi possível aplicar as 
ferramentas de BI no sistema LM. Desta vez, a maioria dos resultados obtidos abordava o tema procurado.

Tabela 1 - Aspectos do protocolo seguido para a R.S.L
Aspectos
Tentativa 1
Tentativa 2

Definição das
palavras chaves

- Business Intelligence

- Lean Manufacturing

Definição das ("Business Intelligence" AND

strings de busca "Lean Manufacturing")
- Business Intelligence

- Decision support tools

- Lean Manufacturing

("Business Intelligence" AND "decision support tools"); (“Lean

Manufacturing" AND "decision support tools")
Critérios de
(I) Artigos de periódicos ou de congressos.
inclusão e
(I) Anos 2008-novembro de 2018.
exclusão
(I) Idiomas: Inglês, Português ou Espanhol.
(I) Inclusão
(E) Artigos duplicados.
(E) Exclusão
(E) Artigos que não contenham nem no Título, Resumo ou Palavras chaves as palavras contidas nas strings de busca.

Fonte: Elaborado pelos autores.

As bases de dados utilizadas foram a Web of Science, Gale One File, Scopus e Science Direct. As duas primeiras bases foram eleitas por conterem os 5 artigos mais relevantes obtidos na primeira busca feita no Portal CAPES, as outras duas pela disponibilidade de estudos publicados relacionados com as engenharias afins.

\section{Execução}

$\mathrm{Na}$ fase de execução são coletados os artigos obtidos das três bases de dados definidas, além da inserção manual de algum artigo de interesse ou referência bibliográfica citada em um dos artigos obtidos das formas anteriores, conhecido como snowbolling. Ao todo foram 53 artigos, a figura 1 apresenta as quantidades de artigos por cada uma das bases de dados. 
Figura 1 - Quantidade da literatura encontrada nas bases de dados

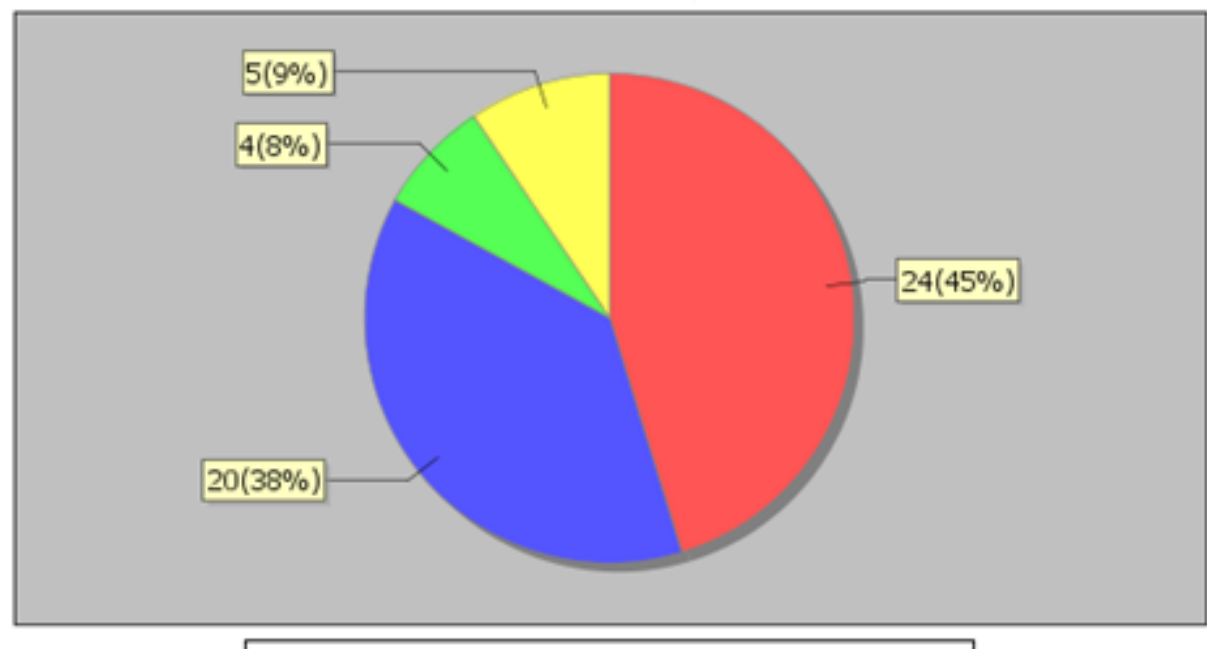

Science Direct - Scopus $\odot$ Web of Science Manually

Fonte: Relatório do StArt.

Logo é feita a seleção dos artigos segundo os critérios de inclusão e exclusão definidos na fase anterior, além dos que estavam duplicados. Na figura 2 são representadas as quantidades de artigos aceitos e rejeitados segundo esses critérios, além dos duplicados.

Aplicando-se os critérios de inclusão serão extraídos os artigos que cumprirem esses critérios e que abordam o tema de principal interesse para a presente pesquisa mediante a leitura mais aprofundada da introdução e conclusão, e caso necessário, leitura do artigo completo.

Figura 2 - Classificação da literatura na análise dos artigos por extenso

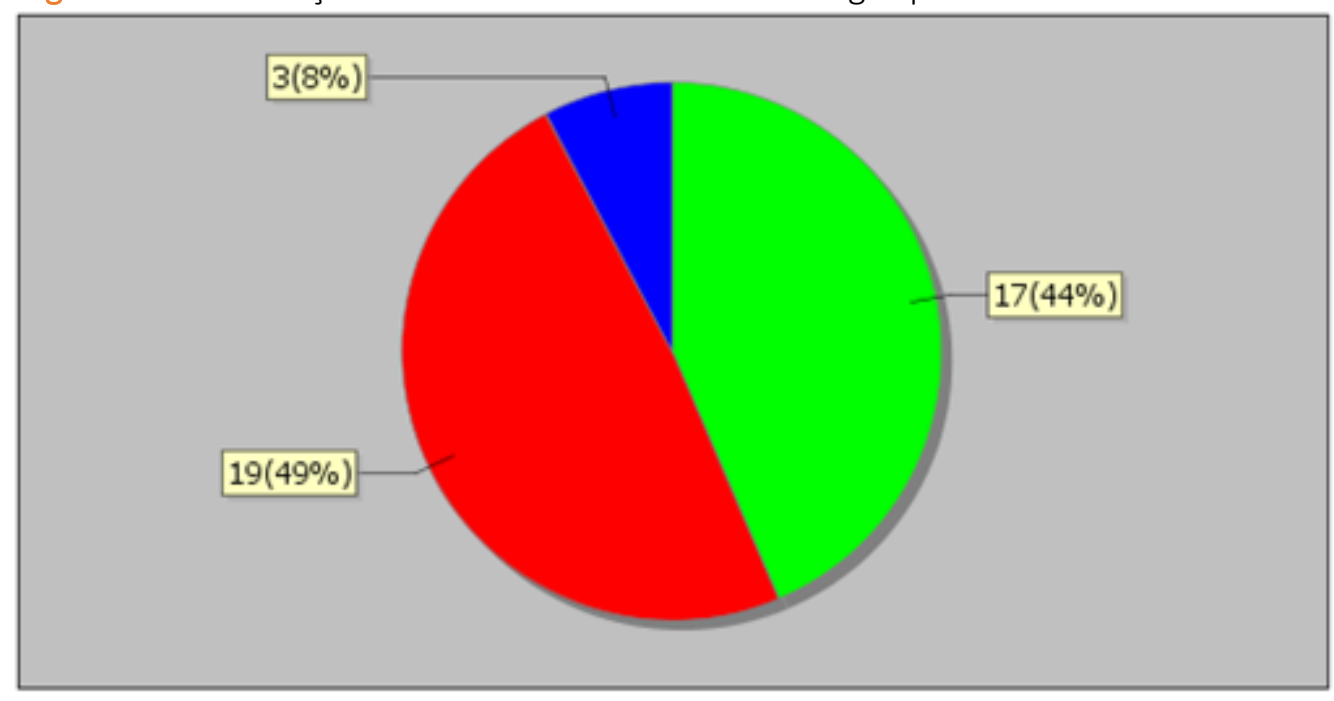

Accepted $\bullet$ Rejected $\bullet$ Duplicated

Fonte: Relatório do StArt.

Assim a análise da literatura foi feita com 17 artigos apresentados no Quadro 1, organizado por ordem decrescente de ano de publicação. 
Quadro 1 - Artigos utilizados para a análise da literatura.

\begin{tabular}{|c|c|c|}
\hline Título da publicação & Autor & Ano \\
\hline $\begin{array}{l}\text { AD-SISCOLO: A decision-support tool to aid the management of a } \\
\text { cervical cancer screening program }\end{array}$ & $\begin{array}{l}\text { Ahmed, Freire,Feitosa, } \\
\text { Zardo \& Almeida }\end{array}$ & 2018 \\
\hline $\begin{array}{l}\text { Business intelligence for performance measurement: A case- } \\
\text { based analysis }\end{array}$ & Vallurupalli \& Bose & 2018 \\
\hline $\begin{array}{l}\text { BI4BI: A continuous evaluation system for Business Intelligence } \\
\text { systems }\end{array}$ & $\begin{array}{l}\text { Brichni, Dupuy-Chessa, } \\
\text { Gzara, Mandran \& Jeannet }\end{array}$ & 2017 \\
\hline $\begin{array}{l}\text { A novel approach to conduct the importance-satisfaction } \\
\text { analysis for acquiring typical user groups in business-intelligence } \\
\text { systems }\end{array}$ & Wang & 2016 \\
\hline $\begin{array}{l}\text { Design and evaluation of hospital-based business intelligence } \\
\text { system (HBIS): A foundation for design science research } \\
\text { methodology }\end{array}$ & $\begin{array}{l}\text { Kao, Yu, Masud, Wu, Chen } \\
\text { \& Wu }\end{array}$ & 2016 \\
\hline Integrated Risk and Quality Management in Hospital Systems & Miniati, Frosini \& Dori & 2016 \\
\hline Cost performance dynamics in lean production leveling & Deif \& Elmaraghy & 2014 \\
\hline Utilizing enterprise systems for managing enterprise risks & Mathrani \& Mathrani & 2013 \\
\hline $\begin{array}{l}\text { An integrated simulation and business intelligence framework for } \\
\text { designing and planning demand responsive transport systems }\end{array}$ & $\begin{array}{l}\text { Telhada, Dias, Sampaio, } \\
\text { Pereira \& Carvalho }\end{array}$ & 2013 \\
\hline Decision support tool for lean product and process development & Pullan, Bhasi \& Madhu & 2013 \\
\hline $\begin{array}{l}\text { Design of an information volatility measure for health care } \\
\text { decision making }\end{array}$ & Tremblay, Hevner \& Berndt & 2012 \\
\hline $\begin{array}{l}\text { Discovering business intelligence from online product reviews: A } \\
\text { rule-induction framework }\end{array}$ & Chung \& Tseng & 2012 \\
\hline $\begin{array}{l}\text { A multi-agent system for web-based risk management in small } \\
\text { and medium business }\end{array}$ & $\begin{array}{l}\text { Bajo, Borrajo, De Paz, } \\
\text { Corchado \& Pellicer }\end{array}$ & 2012 \\
\hline A fuzzy-logic advisory system for lean manufacturing within SMEs & $\begin{array}{l}\text { Achanga, Shehab, Roy \& } \\
\text { Nelder }\end{array}$ & 2012 \\
\hline A tool to evaluate the business intelligence of enterprise systems & Ghazanfari, Jafari \& Rouhani & 2011 \\
\hline Intranets: Web-enabled data warehousing & Murray & 2010 \\
\hline $\begin{array}{l}\text { Decision support for lean practitioners: A web-based adaptive } \\
\text { assessment approach }\end{array}$ & Wan \& Chen & 2009 \\
\hline
\end{tabular}

\section{Sumarização}

Esta última fase é dedicada a análise e resumo dos dados obtidos do estudo dos artigos escolhidos. Foi feita uma análise das palavras chaves como mostrado na figura 3, a palavra com maior frequência de uso dos artigos foi decision-support systems, logo na ordem de maior a menor frequência apareceram as palavras: decision-support tools, business intelligence, risk management, agile- 
manufacturign-systems, artificial intelligence, competitive intelligence, Lean Manufacturing, industry e managers.

Figura 3. Gráfico de nuvem de palavras chaves

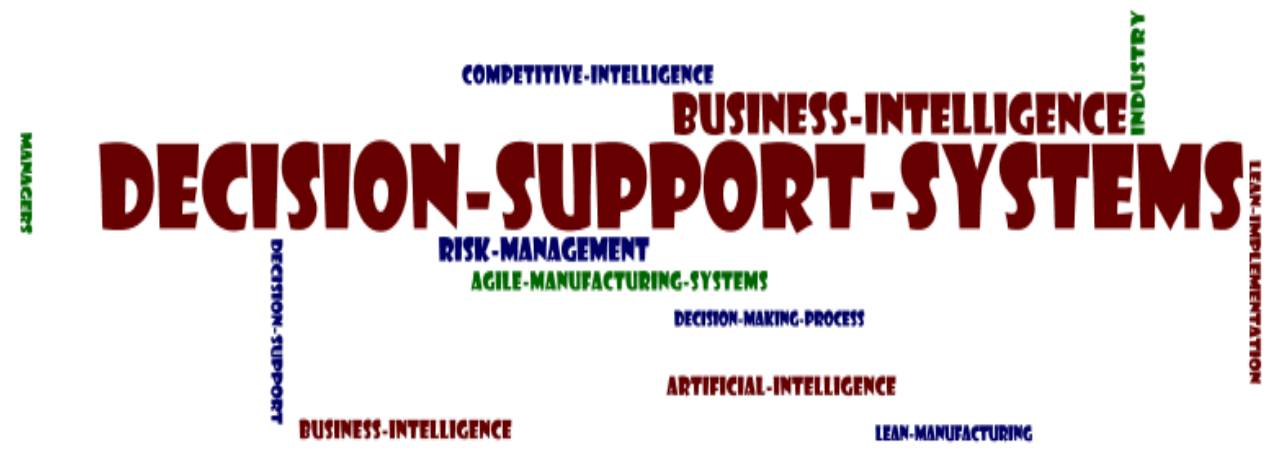

DECISION-SUPPORT-TOOLS

Fonte: Relatório do StArt.

Foi feita uma análise dos autores ao longo dos anos pesquisados, conforme ilustrado na figura 4. Pode-se observar que nos anos de 2009 a 2011, concentram-se a menor quantidade de pesquisas publicadas sobre o tema. O número de pesquisas foi aumentando gradualmente a partir de 2012 até novembro de 2018, o que demonstra o crescente interesse da academia pela área de estudo.

Figura 4 - Gráfico radial de publicações por ano

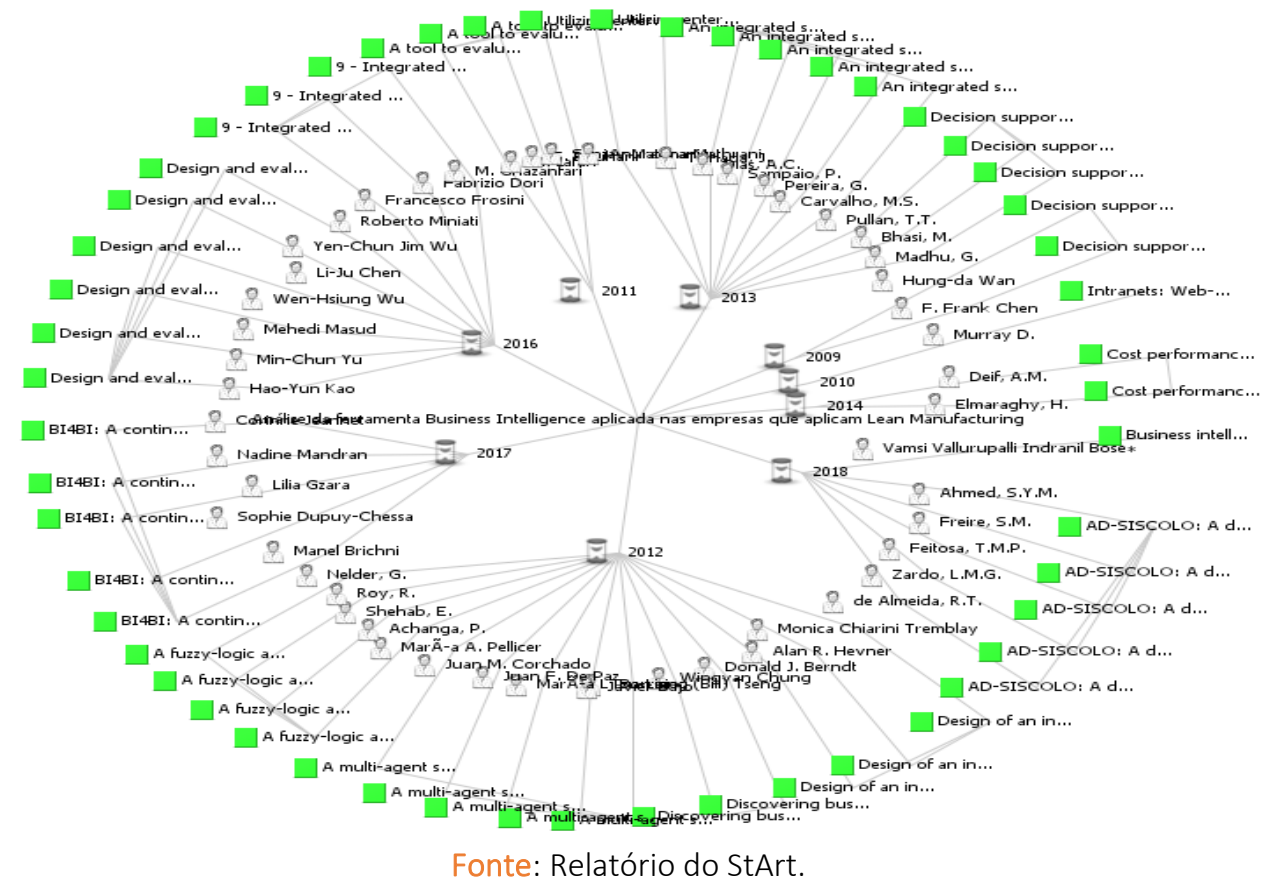




\section{Análise das aplicações de BI como ferramenta de tomada de decisão}

A seguir são apresentadas, no Quadro 2, algumas das aplicações mais recentes do BI como ferramenta de tomada de decisão para diferentes situações e tipos de empresas.

Quadro 2 - Aplicações de BI

Autor/Ano Tipo de aplicação de BI

Ahmed, Freire, Desenvolveram um data warehouse (AD-SISCOLO) com o objetivo de subsidiar a Feitosa, Zardo \& gestão do programa de rastreamento do câncer do colo do útero no município do Rio Almeida, 2018 de Janeiro / Brasil.

Vallurupalli \& Focaram o planejamento, a execução e o gerenciamento de mudanças relacionados Bose, 2018 à implementação de um sistema de medição de desempenho. Procuraram também entender a implementação, uso e impacto do BI, analítico e Big Data nas organizações.

Brichni, Dupuy- Desenvolveram um sistema BI4BI como objetivo de avaliar a análise e a tomada de Chessa, Gzara, decisões sobre a atividade de Bl e sua evolução.

Mandran \&

Jeannet,2017

Kao, Yu, Masud, $\mathrm{Wu}$, Chen \&Wu, 2016

Desenvolveram o sistema inteligente de negócios para hospitais baseado em uma nova metodologia de desenvolvimento, chamada metodologia de pesquisa científica em design e implementado em um hospital geral regional em Taiwan.

Wang, 2016 Propuseram uma nova estrutura para atender aos seguintes objetivos: (1) incorporar as preferências do usuário para identificar os principais recursos de design que melhor se encaixam nos principais segmentos da BI para atingir a aquisição do cliente, (2) transformar as percepções do usuário em graus quantitativos de satisfação e (3) conduzir a análise de importância e satisfação para gerar insights gerenciais para o desenvolvimento de sistemas de BI de próxima geração.

Mathrani e Utilizaram o BI para a eliminação de riscos no nível operacional nas empresas.

Mathrani, 2013

Chung \& Tseng, Desenvolveram uma nova estrutura para projetar sistemas de BI que extraem o 2012 relacionamento entre as avaliações dos clientes e suas revisões. Usando análises de diferentes produtos da Amazon.com, realizaram experimentos qualitativos e quantitativos para avaliar o desempenho de um sistema de BI. 
Mastrapa, L. H., Assumpção, M. R. P. de., \& Campos, F. C. de. (2021, jan./mar.). Business Intelligence + Lean Manufacturing: uma revisão

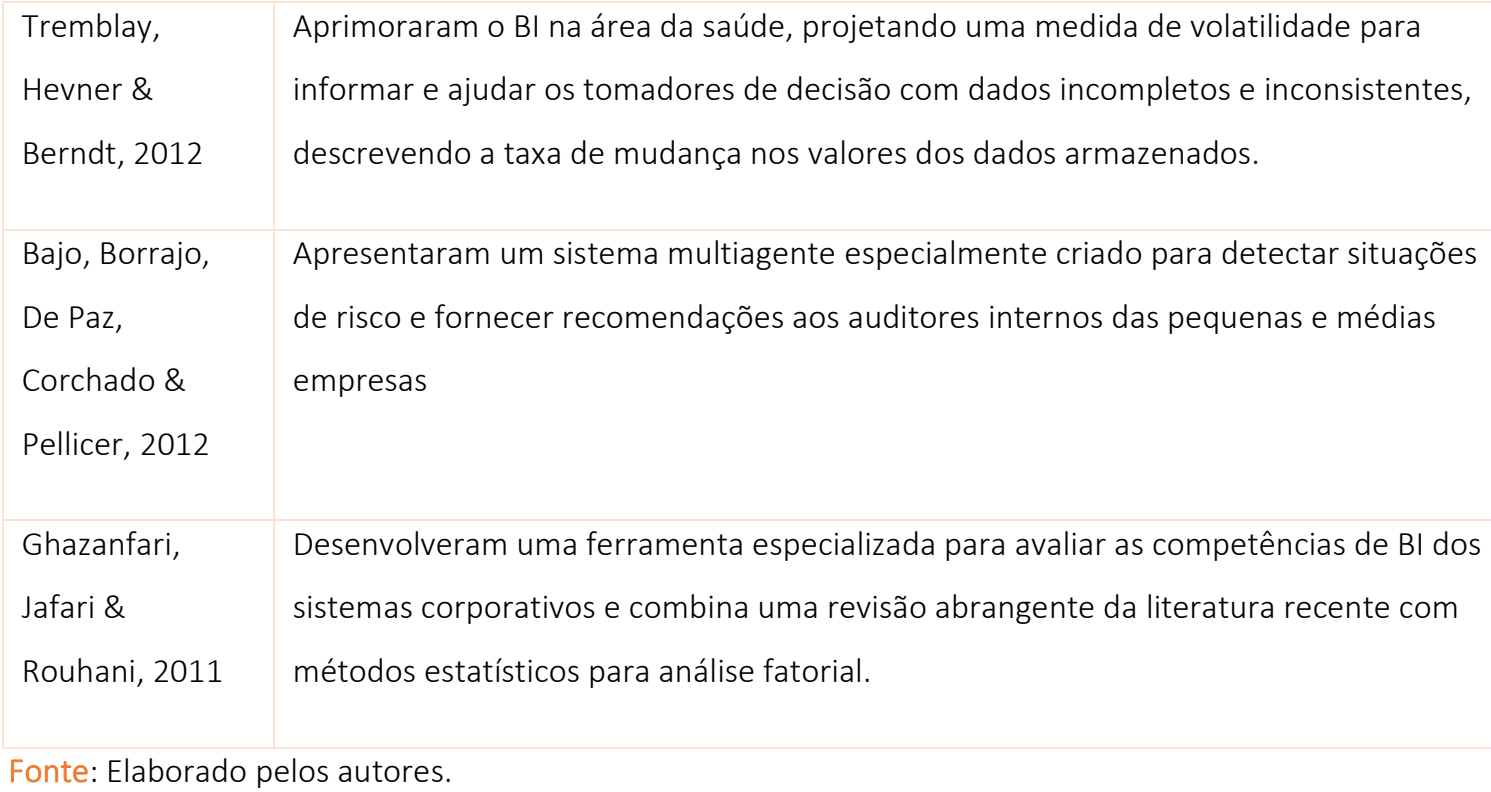

Ahmed, Freire, Feitosa, Zardo \& Almeida (2018), desenvolveram um data warehouse (ADSISCOLO) com o objetivo de subsidiar a gestão do programa de rastreamento do câncer do colo do útero no município do Rio de Janeiro / Brasil. O AD-SISCOLO foi implementado usando a Plataforma de BI do Pentaho BI Suite e o sistema de gerenciamento de banco de dados MYSQL. Os indicadores a serem calculados e visualizados na ferramenta foram baseados nos dados municipais dos exames citopatológico e histopatológico, de janeiro de 2012 a dezembro de 2014, obtidos do Sistema de Informação do Câncer do Colo do Útero (SISCOLO), após um processo de vinculação de registros. Os indicadores de acompanhamento foram baseados em uma versão simplificada das diretrizes brasileiras para o rastreamento do câncer do colo do útero. O AD-SISCOLO permite a visualização de um conjunto de indicadores baseados em testes e de acompanhamento a partir de diferentes visões e dimensões, que permitem aos gerentes monitorar todas as fases do processo de triagem e identificar as falhas do processo.

Vallurupalli \& Bose (2018), oferecem uma estrutura integrada para análise de ponta a ponta da implementação de Sistemas de medição de desempenho (PMS). O framework proposto é holístico, cobrindo todos os aspectos da implementação do PMS: pessoas, processos e tecnologia. O artigo atual é o segundo de uma série de estudos de pesquisa para entender a implementação, uso e impacto do BI, analitics e Big Data nas organizações. O presente documento propõe uma estrutura teoricamente fundamentada para a implementação de um PMS e fornece uma explicação detalhada das diferentes etapas envolvidas na implementação de um PMS baseado em TI. A estrutura proposta no artigo atual é específica e, portanto, mais detalhada e relevante no contexto da implementação do PMS. O atual documento enfocou o planejamento, a execução e o gerenciamento de mudanças relacionados à implementação de um PMS.

Brichni, Dupuy-Chessa, Gzara, Mandran \& Jeannet (2017), apresentaram uma proposta de avaliação contínua de BI. Os autores desenvolveram um sistema BI4BI com o objetivo de avaliar a análise 
e a tomada de decisões sobre a atividade de BI e sua evolução. Inclui duas soluções complementares. Uma solução baseada em sistema depende dos bancos de dados das ferramentas do sistema de BI. Uma solução baseada no usuário depende dos resultados de um questionário que avalia o sistema de BI do ponto de vista de seus usuários. Juntas, as soluções baseadas no sistema e no usuário ajudam a avaliar a atividade do sistema de $\mathrm{BI}$, a fim de garantir sua evolução contínua. O sistema BI4BI foi projetado e desenvolvido em um contexto industrial na STMicroelectronics.

Kao, Yu, Masud, Wu, Chen \&Wu (2016), desenvolveram o sistema inteligente de negócios baseado em hospitais (HBIS- Hospital Business Intelligent System) baseado em uma nova metodologia de desenvolvimento, chamada metodologia de pesquisa científica em design (DSRM-Design Science Research Methodology), e implementado em um hospital geral regional em Taiwan. Uma metodologia de pesquisa em ciência do design é adotada para cobrir seis atividades: identificação e motivação de problemas, definição de objetivos de solução, projeto e desenvolvimento, demonstração, avaliação e comunicação. Com base no método de desenvolvimento do DSRM, o HBIS foi desenvolvido e implantado com sucesso no caso do hospital, e uma pesquisa com usuários mostra resultados positivos. O sistema integra e fornece fácil acesso a indicadores de gestão médica, permitindo que administradores e profissionais aprimorem a correção e a pontualidade das ações corretivas, reduzindo assim os problemas e aumentando a eficiência geral.

Wang (2016), propuseram uma nova estrutura para atender aos seguintes objetivos: (1) incorporar as preferências do usuário para identificar os principais recursos de design que melhor se encaixam nos principais segmentos da BI para atingir a aquisição do cliente, (2) transformar as percepções do usuário em graus quantitativos de satisfação e (3) conduzir a análise de importância e satisfação para gerar insights gerenciais para o desenvolvimento de sistemas de BI de próxima geração.

Mathrani \& Mathrani (2013), O gerenciamento de riscos corporativos é um conceito crítico no atual ambiente de negócios que suporta o uso de ferramentas e processos voltados para o monitoramento e a mitigação de riscos organizacionais. Muitas organizações adotaram a tecnologia de sistemas empresariais (ESs) para melhorar a eficiência e a eficácia organizacional. No nível executivo, o uso do módulo de BI ajudou a avaliar o progresso para atingir as metas e as traduziu em ações de eliminação de riscos no nível operacional. Uma abordagem inicial de impulso de tecnologia ajudou a criar modelos de processos representativos semanticamente ricos, simulando cenários de risco, levando a uma abordagem de estratégia para implementar estratégias e decisões de negócios. Um modelo estratégico de avaliação de valor articula os processos de alavancagem de conhecimento combinando habilidades humanas com ferramentas ESs para otimizar os riscos da empresa. Os resultados indicam que o processo de transformação de dados de ESs resultou de ações de alavancagem de conhecimento nos níveis executivo e operacional.

Chung \& Tseng (2012), desenvolveram de uma nova classe de sistemas de BI baseados em teoria de conjuntos aproximados, aprendizado de regras indutivas e métodos de recuperação de informações. 
Essa nova estrutura extraem o relacionamento entre as avaliações dos clientes e suas revisões. Usando análises de diferentes produtos da Amazon.com, realizaram experimentos qualitativos e quantitativos para avaliar o desempenho de um sistema de BI desenvolvido com base na estrutura. Os resultados indicam que o sistema alcançou alta precisão e cobertura relacionadas à qualidade das regras e produziu regras interessantes e informativas com altos valores de suporte e confiança. As descobertas têm importantes implicações para a análise do comportamento do mercado e o gerenciamento da reputação do comércio eletrônico. Tremblay, Hevner \& Berndt (2012), sob a preocupação da instabilidade dos dados subjacentes para estimativas pontuais e tendências usadas em ferramentas que apresentam dados agregados, como OLAP (Online Analytical Processing), planilha ou ferramentas de relatório, na área da saúde, projetaram uma medida de volatilidade da informação. Utilizando métodos de pesquisa do design science, os autores projetaram esta medida de volatilidade para informar e ajudar os tomadores de decisão com dados incompletos e inconsistentes, descrevendo a taxa de mudança nos valores dos dados armazenados. O cálculo é baseado na distribuição subjacente e baseia-se em pesquisas anteriores em finanças, uma área que examina o risco e o comportamento futuro usando dados que compartilham semelhanças com os dados encontrados nas cadeias de fornecimento de informações de assistência médica.

Bajo, Borrajo, De Paz, Corchado \& Pellicer (2012), apresentaram um sistema multiagente especialmente criado para detectar situações de risco e fornecer recomendações aos auditores internos das pequenas e médias empresas (PMEs). O núcleo do sistema multiagente é um tipo de agente com capacidades avançadas de raciocínio para fazer previsões baseadas em experiências anteriores. Esse tipo de agente é usado para implementar um agente avaliador especializado em detectar situações de risco e um agente orientador destinado a fornecer recursos de suporte à decisão. Ambos os agentes incorporam técnicas inovadoras que pode contribuir para detectar potenciais situações de risco e evitálas, agindo nas tarefas que compõem cada uma das atividades do negócio.

Ghazanfari, Jafari \& Rouhani (2011), desenvolveram uma ferramenta especializada para avaliar as competências de $\mathrm{BI}$ dos sistemas corporativos e combina uma revisão abrangente da literatura recente com métodos estatísticos para análise fatorial. Uma análise estatística identificou seis fatores para o modelo de avaliação: "Apoio analítico e inteligente à decisão", "Fornecimento de experimentos e integração relacionados com informações ambientais", "Otimização e modelo recomendado", "Raciocínio", "Ferramenta de tomada de decisão aprimorada" e, finalmente, "Satisfação dos stakeholders". Utilizando as cargas extraídas de cada critério único, a inteligência dos sistemas de trabalho pode ser medida e representada em seis painéis, com base em fatores correspondentes, atualizando uma ferramenta especializada que pode diagnosticar o nível de inteligência dos sistemas corporativos. As empresas podem usar essa abordagem para avaliar, selecionar e comprar software e sistemas que ofereçam melhor suporte a decisões para o ambiente organizacional, permitindo que eles obtenham vantagem competitiva. 
Análise das ferramentas de tomada de decisão utilizadas nas empresas que aplicam o sistema Lean Manufacturing

Nesta seção são apresentadas, no Quadro 3, algumas das ferramentas para a tomada de decisão nas empresas que aplicam o sistema LM, com o intuito de analisar as possíveis semelhanças e ou oportunidades de aplicações com as ferramentas de Bl.

Quadro 3 - Aplicações de ferramentas de tomada de decisão nas empresas LM

Wan \& Chen, 2009

Desenvolveram uma ferramenta de apoio à decisão baseada na web usando a abordagem de avaliação adaptativa como uma ferramenta de orientação do processo de implementação enxuta.

Achanga, Shehab, Roy \&

Nelder, 2012

Pullan, Bhasi \& Madhu, 2013

Deif \& Elmaraghy, 2014
Apresentaram o desenvolvimento de um sistema de consultoria de lógica fuzzy para auxiliar pequenas e médias empresas como uma ferramenta de apoio à decisão para implementar a manufatura enxuta.

Propuseram uma estrutura de engenharia enxuta e simultânea baseado na aplicação de tecnologia da informação e metodologia orientada a objeto para manufatura enxuta. Um estudo de caso é apresentado em uma empresa fabricante de máquinas-ferramenta. A prática de Engenharia enxuta e simultânea foi adotada para novos produtos, para alinhar os sistemas e processos da empresa. Propuseram uma abordagem de sistemas dinâmicos para investigar os desafios da implementação do nivelamento da produção e dos custos associados. O modelo captura várias ferramentas enxutas que influenciam o nivelamento da produção e suas implicações. Análises de custo comparativos entre várias políticas de implementação de nivelamento para demanda estocástica com múltiplos produtos são conduzidas.

Fonte: Elaborado pelos autores.

Wan \& Chen (2009), apresentaram uma abordagem de avaliação enxuta adaptativa que fornece uma maneira eficaz de guiar o processo de implementação enxuta. Usando o programa baseado na web, um modelo de avaliação é gerado de forma adaptativa para cada usuário avaliar o status atual do sistema, identificar as metas urgentes de melhoria e identificar as ferramentas e técnicas apropriadas para o desenvolvimento de planos de ação. A fim de fornecer suporte suficiente para os profissionais enxutos, a ferramenta gera tanto escores enxutos como diretrizes de melhoria adaptativa de acordo 
com o tipo e as condições atuais do sistema pesquisado. Usando os relatórios, os usuários podem identificar as ferramentas corretas e aplicá-las no momento certo, no ponto certo do sistema. Um programa baseado na web foi desenvolvido para executar a ferramenta proposta. Os resultados preliminares demonstram uma nova contribuição no uso de ferramentas de suporte a decisão para a manufatura enxuta.

Achanga, Shehab, Roy \& Nelder (2012), apresentaram o desenvolvimento de um sistema de consultoria de lógica difusa para auxiliar PMEs como uma ferramenta de apoio à decisão para implementar a manufatura enxuta. O sistema é desenvolvido usando regras de lógica fuzzy, com uma combinação de abordagens de metodologia de pesquisa empregadas no estudo que incluiu coleta de dados de 10 PMEs de manufatura por meio de análise de documentação, observação de práticas de empresas e entrevistas semi-estruturadas. O sistema global compreende três subsistemas de consultoria de lógica difusa que alimentam um sistema principal. Esses resultados são o custo relativo da implementação enxuta, um status de prontidão enxuta da empresa e o nível de agregação de valor a ser alcançado (impacto / benefícios). O sistema desenvolvido fornece regras heurísticas que auxiliam o processo de tomada de decisão para implementação enxuta no estágio inicial de implementação. A facilidade de visualização do sistema desenvolvido também é uma ferramenta útil para permitir que os usuários enxutos em potencial possam prever antecipadamente o custo relativo do projeto enxuto, antecipar os benefícios enxutos e perceber o grau de prontidão enxuta.

Segundo Pullan, Bhasi \& Madhu (2013) as estratégias de engenharia enxuta e simultânea podem melhorar os processos, reduzir custos e reduzir o desperdício, permitindo que as organizações permaneçam competitivas. Este artigo propõe uma estrutura de engenharia enxuta e simultânea baseado na aplicação de tecnologia da informação e metodologia orientada a objeto para manufatura enxuta. Um estudo de caso é apresentado neste artigo para demonstrar a eficácia da estrutura de engenharia enxuta e simultânea em uma empresa fabricante de máquinas-ferramenta. A prática da engenharia enxuta e simultânea foi adotada para novos produtos, para alinhar os sistemas e processos da empresa. O lead time de desenvolvimento de produtos diminuiu em mais de 50\% em comparação com projetos de desenvolvimento similares realizados pela empresa. A necessidade de retrabalho foi considerada insignificante e o custo de desenvolvimento foi reduzido consideravelmente.

Deif \& Elmaraghy (2014), propuseram uma abordagem de sistemas dinâmicos para investigar os desafios da implementação do nivelamento da produção e dos custos associados. O modelo captura várias ferramentas enxutas que influenciam o nivelamento da produção e suas implicações. Analisam e comparam o custo entre várias políticas de implementação de nivelamento para demanda estocástica com múltiplos produtos são conduzidas. O modelo desenvolvido e os insights obtidos com os resultados podem ajudar os profissionais de manufatura enxuta a decidir melhor quando e como implementar o nivelamento da produção, bem como determinar os tamanhos e a sequência dos lotes de produção. Eles também enfatizam a importância da análise de custos como ferramenta auxiliar de apoio à decisão 
no trade-off exigido entre os benefícios de diferentes níveis de políticas enxutas e seu custo associado. Esta pesquisa demonstra que os benefícios obtidos pela implementação de políticas enxutas em sistemas de manufatura dependem de muitos sistemas e de fatores relacionados ao mercado. O modelo de dinâmica do sistema apresentado fornece suporte para tomadores de decisão e ajuda a explorar muitos cenários hipotéticos e custos e benefícios associados.

\section{Possíveis aplicações da ferramenta BI nas empresas que aplicam o Sistema LM. Análise SWOT}

A análise da literatura apresentada nas seções anteriores permitiu observar o desempenho das ferramentas utilizadas para a tomada de decisão nas empresas que aplicam o sistema LM, assim como as aplicações, nos diferentes setores empresariais, das ferramentas de BI.

A análise da literatura relacionada com as aplicações das ferramentas de BI, permitiu determinar a funcionalidade desta ferramenta, devido a aplicabilidade que ela possui nos diferentes setores empresariais e áreas das empresas. Segundo o estudo da literatura relacionada com as ferramentas de tomada de decisão aplicadas às empresas que implementam o LM, a maioria dos casos analisados referem-se a tomada de decisão de aplicar ou aprimorar indicadores do sistema LM. Dos casos analisados, segundo a descrição das ferramentas aplicadas, existem semelhanças com a ferramenta $\mathrm{Bl}$, sendo que a maioria destes casos procuram coletar dados e transformá-los em informações para a posterior tomada de decisão. Nenhum destes estudos aborda de forma explicita a possibilidade de usar a ferramenta de $\mathrm{Bl}$, mas, pela aplicabilidade que esta ferramenta possui, pode-se inferir que ela poderia ser uma boa estratégia para o apoio na tomada de decisão para as empresas que implementam o LM ou desejam implementá-lo proximamente. A modo de resumo, é apresentado a seguir, no Quadro 4, uma análise SWOT (Strengths, Weaknesses, Opportunities e Threats) com o intuito de apresentar os pontos fortes e pontos fracos da ferramenta BI e do sistema de manufatura enxuta na tomada de decisão assim como as oportunidades e ameaças da aplicação das ferramentas de BI para o sistema de manufatura enxuta. 
Quadro 4 - Análise SWOT das ferramentas de BI e LM

\begin{tabular}{|c|c|}
\hline 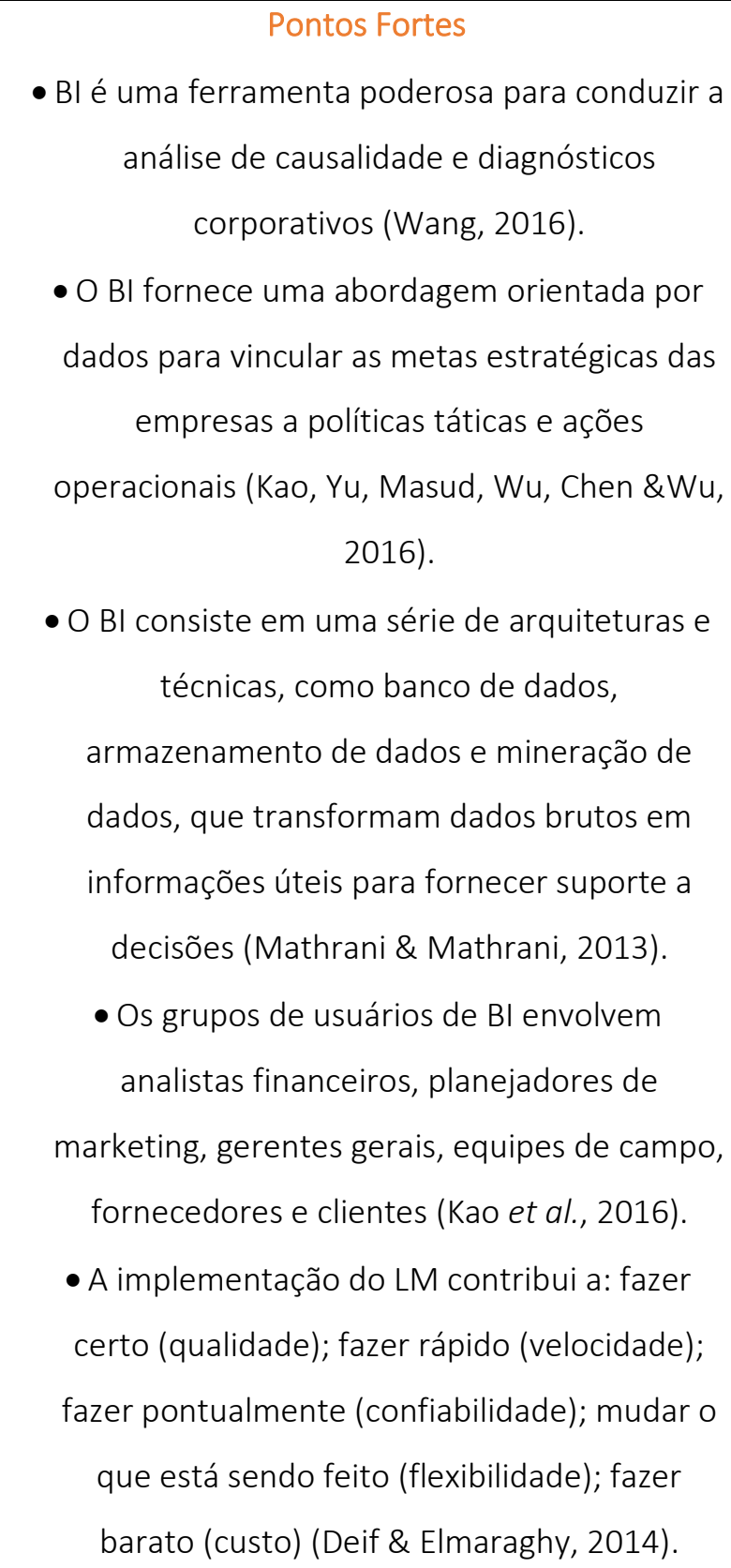 & $\begin{array}{c}\text { Pontos Fracos } \\
\text { - O BI pode gerar despesas } \\
\text { adicionais relacionadas à } \\
\text { infraestrutura; Aprendizado lento } \\
\text { (usuários); Baixo Retorno sobre o } \\
\text { Investimento (ROI) (Kao, Yu, } \\
\text { Masud, Wu, Chen \&Wu, 2016). } \\
\text { - A implementação do LM pode } \\
\text { gerar altos custos em sua fase } \\
\text { inicial; assim como restrição da } \\
\text { variabilidade de produtos (Deif \& } \\
\text { Elmaraghy, 2014). }\end{array}$ \\
\hline $\begin{array}{c}\text { Oportunidades } \\
\text { Viabilidade da utilização dos conceitos de BI/ Data } \\
\text { warehouse para o desenvolvimento e } \\
\text { gerenciamento de indicadores de manufatura } \\
\text { enxuta (Escodeiro \& Pereira, 2009). }\end{array}$ & $\begin{array}{c}\text { Ameaças } \\
\text { - Falta de foco (insucesso após a } \\
\text { implementação) (Vallurupalli \& } \\
\text { Bose, 2018). } \\
\text { • Pouca literatura que relate } \\
\text { experiências de aplicações de BI } \\
\text { em empresas que apliquem o } \\
\text { sistema de manufatura enxuta. }\end{array}$ \\
\hline
\end{tabular}

Fonte: Elaborado pelos autores. 
Os pontos fortes descritos demonstram o potencial que tanto a ferramenta BI quanto o sistema LM fornecem as empresas. Dentro das ameaças a falta de foco ou insucesso após a implementação (Vallurupalli \& Bose, 2018), assim como a pouca literatura que relate experiências de aplicações de BI em empresas que apliquem o sistema de manufatura enxuta, observado pelos autores na presente pesquisa, pode atentar contra o interesse de aplicação por parte das empresas. Mas a viabilidade da utilização dos conceitos de BI/ Data warehouse para o desenvolvimento e gerenciamento de indicadores de manufatura enxuta constitui uma oportunidade de aplicação acertada que, como consequência, geraria grandes vantagens as empresas além do apoio à tomada de decisão.

\section{Considerações finais}

A busca feita nas bases de dados, não obteve nenhum resultado em que a ferramenta de $\mathrm{BI}$ fosse aplicada para a tomada de decisão em empresas que aplicam o LM ou que estejam no processo de implantá-lo. Esta constatação pode ser verificada nos quadros da síntese dos principais trabalhos encontrados nesta temática. Com isto, a fim de encontrar oportunidades de aplicação da ferramenta BI no contexto LM, e cumprir com o objetivo do presente estudo, foi feita a busca e análise de forma separada como apresentada nas seções anteriores. Isto permitiu observar o desempenho das ferramentas utilizadas para a tomada de decisão nas empresas que aplicam o LM, assim como as aplicações, nos diferentes setores empresariais, das ferramentas de BI. Foi feita uma análise SWOT com o intuito de apresentar os pontos fracos e oportunidades que possibilitam a aplicação de BI para o sistema de LM assim como as fraquezas e ameaças que podem inibi-la.

Finalmente, o estudo feito não identificou nenhuma característica particular que impedisse a aplicação da ferramenta de BI no LM, considerando que existe potencial para isso.

A aplicação da ferramenta de BI em uma empresa que implantou LM para a tomada de decisão é sugerida como trabalho futuro. Além disso, este estudo pode ser um ponto de partida para futuras pesquisas dedicadas a análise da implementação da ferramenta BI no contexto LM.

\section{Agradecimentos:}

O presente trabalho foi realizado com apoio da Coordenação de Aperfeiçoamento de Pessoal de Nível Superior - Brasil (CAPES) - Código de Financiamento 001.

\section{Referências}

Achanga, P., Shehab, E., Roy, R. \& Nelder, G. (2012) A fuzzy-logic advisory system for lean manufacturing within SMEs, International Journal of Computer Integrated Manufacturing, 25(9), pp. 839-852. 
Ahmed, S., Freire, S., Feitosa, T., Zardo, L. \&Almeida, R. (2018) AD-SISCOLO: a decision-support tool to aid the management of a cervical cancer screening program, Research on Biomedical Engineering, 34(1), pp. 19-30.

Bajo, J., Borrajo, M. L., De Paz, J. F., Corchado, J. M. \& Pellicer, M. A. (2012) A multi-agent system for web-based risk management in small and medium business, Expert Systems with Applications. Elsevier Ltd, 39(8), pp. 6921-6931.

Brichni, M. Dupuy-Chessa, S., Gzara, L., Mandran, N. \& Jeannet, C. (2017) BI4BI: A continuous evaluation system for Business Intelligence systems, Expert Systems with Applications. Elsevier Ltd, 76, pp. 97-112.

Chen, H., Chiang, R. H. \& Storey, V. C. (2012) Business intelligence and analytics:from big data to big impact.Mis Quarterly, 36(4), 1165-1188.

Chung, W. \& Tseng, T. L. (2012) Discovering business intelligence from online product reviews: A ruleinduction framework, Expert Systems with Applications. Elsevier Ltd, 39(15), pp. 11870-11879.

Deif, A. M. \& Elmaraghy, H. (2014) Cost performance dynamics in lean production leveling, Journal of Manufacturing Systems. The Society of Manufacturing Engineers, 33(4), pp. 613-623.

Escodeiro, J. R. \& Pereira, N. A. (2009) Desenvolvimento De Indicadores Da Manufatura Enxuta Utilizando Ferramentas De Business Intelligence: Uma Aplicação Na Manufatura De Calçados, UFSCar - Universidade Federão de São Carlos, p. 162.

Fabbri, S., Silva, C., Hernandes, E., Octaviano, F., Di Thommazo, A. \&Belgamo, A. (2016) Improvements in the StArt tool to better support the systematic review process, Proceedings of the 20th International Conference on Evaluation and Assessment in Software Engineering - EASE '16, pp. 15.

Ghazanfari, M., Jafari, M. \& Rouhani, S. (2011) A tool to evaluate the business intelligence of enterprise systems, Scientia Iranica. Elsevier B.V., 18(6), pp. 1579-1590.

Ghosh, B., \& Scott, J. E. (2011). Antecedents and catalysts for developing a healthcareanalytic capability. Communications of the Association for Information Systems,29(1), 395-409.

Haque, W., Derksen, B. A., Calado, D., \& Foster, L. (2015). Using business intelligencefor efficient interfacility patient transfer.Stud Health Technol Inform, 208,170 -176.

Kao, H. Y.,Yu, M., Masud, M., Wu, W., Chen, L. \& Wu, Y. (2016) Design and evaluation of hospitalbased business intelligence system (HBIS): A foundation for design science research methodology, Computers in Human Behavior. Elsevier Ltd, 62, pp. 495-505.

Kitchenham, B. \& Charters, S. 2007. Guidelines for Performing Systematic Literature Reviews in Software Engineering. Technical Report. Keele University and University of Durham, version 2.3.

Mathrani, S. \& Mathrani, A. (2013) 'Utilizing enterprise systems for managing enterprise risks', Computers in Industry. Elsevier B.V., 64(4), pp. 476-483.

Miniati, R., Frosini, F., \& Dori, F. (2016). Integrated Risk and Quality Management in Hospital Systems. Clinical Engineering, 117-130.

Murray D. (2010) ' Intranets: Web-enabled data warehousing', The CRC Handbook of Modern Telecommunications. 
Negash, S. (2004). Business intelligence.Communications of the Association for In-formation Systems, 13(1), 177e195.

Pereira, M. G. \& Galvão, T. F. (2014) 'Etapas de busca e seleção de artigos em revisões sistemáticas da literatura', Epidemiologia e Serviços de Saúde, 23(2), pp. 369-371.

Pullan, T. T., Bhasi, M. \& Madhu, G. (2013) 'Decision support tool for lean product and process development', Production Planning and Control, 24(6), pp. 449-464.

Shollo, A., \& Galliers, R. D. (2015). Towards an understanding of the role of businessintelligence systems in organisational knowing.Information Systems Journal.

Taddeo, R., Simboli, A., Di Vincenzo, F. \& Ioppolo, G. (2019) 'A bibliometric and network analysis of Lean and Clean(er) production research (1990/2017)', Science of the Total Environment. Elsevier B.V., 653, pp. 765-775.

Telhada, J., Dias, A. C., Sampaio, P., Pereira, G., \& Carvalho, M. S. (2013). An Integrated Simulation and Business Intelligence Framework for Designing and Planning Demand Responsive Transport Systems. Computational Logistics, 98-112.

Tremblay, M. C., Hevner, A. R. \& Berndt, D. J. (2012) 'Design of an information volatility measure for health care decision making', Decision Support Systems. Elsevier B.V., 52(2), pp. 331-341. oi: 10.1016/j.dss.2011.08.009.

Vallurupalli, V. \& Bose, I. (2018) 'Business intelligence for performance measurement: A case based analysis', Decision Support Systems. Elsevier, 111(May), pp. 72-85.

Wan, H. \& Chen, F. F. (2009) 'Decision support for lean practitioners: A web-based adaptive assessment approach', Computers in Industry, 60(4), pp. 277-283.

Wang, C. H. (2016) 'A novel approach to conduct the importance-satisfaction analysis for acquiring typical user groups in business-intelligence systems', Computers in Human Behavior. Elsevier Ltd, 54, pp. 673-681. 